sequencing-based diversity studies. Nucleic Acids Res. 2013;41:e1. https://doi.org/10.1093/nar/gks808

7. Callahan BJ, McMurdie PJ, Rosen MJ, Han AW, Johnson AJA, Holmes SP. DADA2: high-resolution sample inference from Illumina amplicon data. Nat Methods. 2016;13:581-3. https://doi.org/10.1038/nmeth.3869

8. Mediannikov O, Sekeyová Z, Birg M-L, Raoult D. A novel obligate intracellular gamma-proteobacterium associated with ixodid ticks, Diplorickettsia massiliensis, gen. nov., sp. nov. PLoS One. 2010;5:e11478. https://doi.org/10.1371/ journal.pone.0011478

9. Subramanian G, Mediannikov O, Angelakis E, Socolovschi C, Kaplanski G, Martzolff L, et al. Diplorickettsia massiliensis as a human pathogen. Eur J Clin Microbiol Infect Dis. 2012;31: 365-9. https://doi.org/10.1007/s10096-011-1318-7

Address for correspondence: David Allen, Department of Biology, Middlebury College, McCardell Bicentennial Hall 372, Middlebury, VT 05753, USA; email: dallen@middlebury.edu

\section{Case of Babesia crassa-Like Infection, Slovenia, 2014}

\author{
Katja Strasek-Smrdel, Misa Korva, Emil Pal, \\ Mojca Rajter, Miha Skvarc, Tatjana Avsic-Zupanc
}

\begin{abstract}
Author affiliations: Institute of Microbiology and Immunology, Faculty of Medicine, University of Slovenia, Ljubljana, Slovenia (K. Strasek-Smrdel, M. Korva, M. Skvarc, T. Avsic-Zupanc); Murska Sobota General Hospital, Rakican, Slovenia (E. Pal); University Medical Centre Ljubljana, Ljubljana (M. Rajter)
\end{abstract}

DOI: https://doi.org/10.3201/eid2605.191201

We report a case of Babesia crassa-like infection in an asplenic patient in Slovenia in 2014. We diagnosed the infection using microscopy, 18S rRNA sequencing, and serology and monitored parasitemia using digital PCR. With its increasing occurrence, babesiosis should be included in differential diagnoses for immunocompromised patients displaying fever.

B abesia infections occur worldwide and cause disease mainly in animals, but disease occurs occasionally in humans. Infections in humans are mostly attributable to B. microti, B. duncani, and Babesia sp. MO1 in North America; B. divergens, B. venatorum, and B. microti in Europe; and B. venatorum, B. crassalike parasite, B. microti, Babesia sp. XXB/HangZhou, and Babesia sp. KO-1 in Asia $(1,2)$. Transmission occurs predominantly through tick bites, but humans have acquired infections via contaminated blood products and through the transplacental and perinatal routes (1). Most patients with Babesia infections in Europe were reported to be asplenic or immunocompromised. Typical clinical signs and symptoms include fever (up to $40^{\circ} \mathrm{C}$ ), parasitemia $(20 \%-80 \%)$, severe anemia, muscle weakness, fatigue, and lateonset jaundice with dark urine, and sometimes complications develop. Long-term clinical follow-up that includes blood smear examination and PCR analysis is necessary because relapse and persistence of parasitemia can occur in spite of treatment. The application of novel molecular methods has revealed that the host range of many Babesia species is less restricted than previously thought. New species or animal pathogens are increasingly being discovered as causing Babesia infections in humans. We report a B. crassa-like infection in a patient in Slovenia in 2014.

In 2014, a 55-year-old woman, living on the outskirts of Murska Sobota, Slovenia, sought medical treatment for a 6-day history of intermittent fever up to $39^{\circ} \mathrm{C}$, myalgia, headache, poor appetite concomitant with weight loss, fatigue, sweating, and dark urine. She previously had a splenectomy and partial pancreatectomy 5 years previous because of cystic adenoma and adrenal incidentaloma without hormonal activity. She reported no history of travel, tick bite, animal contact, or blood transfusions.

Her blood pressure was 115/70 $\mathrm{mm} \mathrm{Hg}$, heart rate 83 beats/min, and body temperature $36.6^{\circ} \mathrm{C}$, and a physical examination indicated no significant clinical findings. The first basic blood analysis revealed thrombocytopenia (platelets $85 \times 10^{9} / \mathrm{L}$ ). A differential blood analysis indicated that the concentration of large unstained cells was elevated $(0.41$ $\times 10^{9} / \mathrm{L}$, reference range $0-0.4 \times 10^{6} / \mathrm{L}$ ). Biochemical laboratory testing showed mild fluctuations in liver functioning: aspartate aminotransferase 1.22 (reference range 0.17-0.51) $\mu \mathrm{kat} / \mathrm{L}$, alanine aminotransferase 1.13 (reference range 0.17-0.68) $\mu \mathrm{kat} / \mathrm{L}$, $\gamma$-glutamyltransferase 1.08 (reference range 0.03-0.51 $\mu \mathrm{kat} / \mathrm{L}$ ) $\mu \mathrm{kat} / \mathrm{L}$, and alkaline phosphatase 1.88 (reference range 0.5-2.0) $\mu \mathrm{kat} / \mathrm{L}$. C-reactive protein was $51 \mathrm{mg} / \mathrm{L}$ (150 [reference range 0.76-28.5] nmol/L), and mild erythrocyturia was present. Giemsa-stained blood smears showed unusual inclusions in erythrocytes, Howell-Jolly bodies, mild anisocytosis, some atypical lymphocytes, and some large thrombocytes. We observed many ring forms and some paired piriform shapes of Babesia spp. in blood smears (Figure), and parasitemia was 1\% (Appendix Table, 

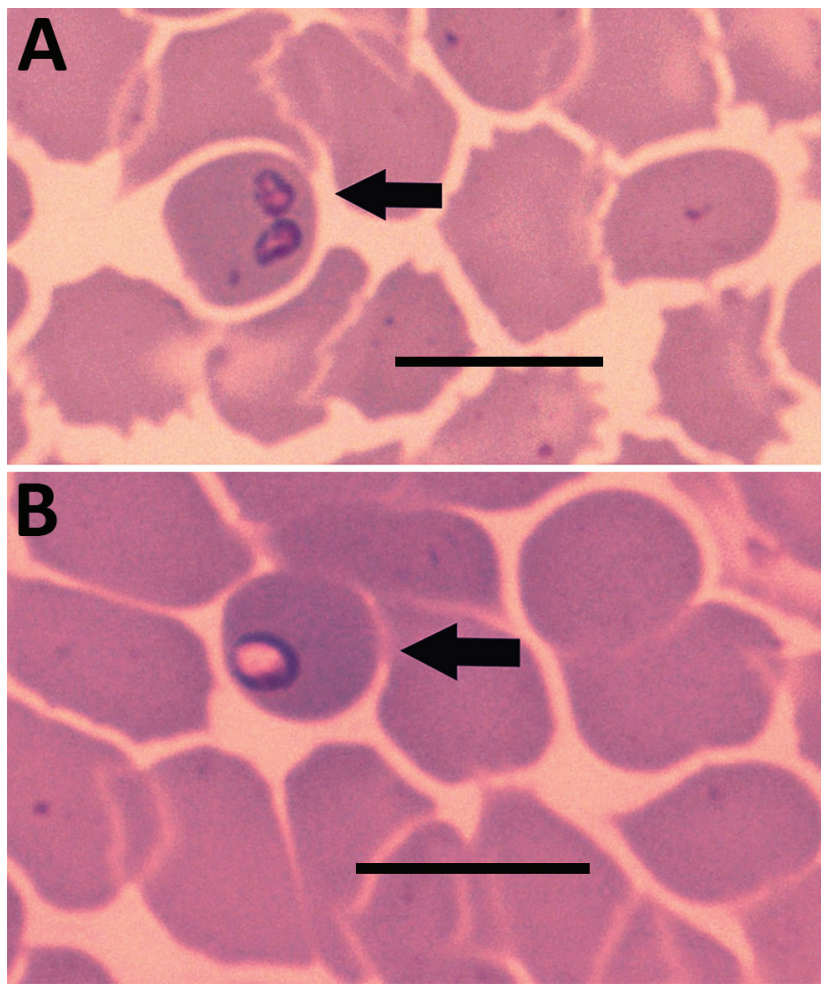

Figure. Piriform (A) and ring shapes $(B)$ in blood smear of sample taken from patient with Babesia crassa-like infection, Slovenia, 2014. Smear was Wright-Giemsa stained. Scale bars indicate $50 \mu \mathrm{m}$.

https://wwwnc.cdc.gov/EID/article/26/5/19-1201App1.pdf). We confirmed diagnosis by conventional PCR and sequencing of the $18 \mathrm{~S}$ rRNA gene (3). A phylogenetic analysis indicated the pathogen was the $B$. crassa-like parasite (Appendix Figure).

We gave the patient an oral treatment of clindamycin (600 $\mathrm{mg} 3 \times / \mathrm{d})$ and quinine $(600 \mathrm{mg}$ $3 \times / d)$. Three days later, the patient was normothermic, and after a total of 6 days, she was discharged from the hospital with platelet levels within the reference range $\left(150-350 \times 10^{9} / \mathrm{L}\right)$. She continued the dual therapy for 14 days. To follow up on the patient's response to treatment, we measured parasitemia levels by blood smear microscopy, PCR (3), and digital PCR (Appendix).

We later confirmed the infection by serology using an indirect immunofluorescence assay specific to another member of the large Babesia group, B. divergens (MegaFLUO BABESIA divergens; Megacor, https://www.megacor.at). Antibodies were crossreactive, and results demonstrated a 4 -fold increase in IgG titer (Appendix Table).

Reports of babesiosis in humans are increasing with the increase in number of immunocompromised persons; a species previously known only as an animal pathogen is posing a greater threat to those with weakened immune systems. $B$. crassa has been detected in sheep in Iran (4), goats and ticks in Turkey $(5,6)$, and ticks in Hungary (7), and a case series of infections with $B$. crassa-like parasite in humans, sheep, and ticks was reported in northeastern China (8).

We report an infection of $B$. crassa-like parasite in an asplenic person in Europe that was confirmed by blood smear examination, PCR, sequencing, and serology (with assay specific to distant relative $B$. divergens). The patient recovered after treatment with the standard dual antimicrobial regimen. In addition to blood smear, we used a unique digital PCR assay to follow the decrease in concentration of babesial DNA in the patient's blood until complete recovery. Note that DNA levels in blood do not necessarily correlate with levels of live pathogen (i.e., active infection).

With the development of new and more sensitive diagnostic techniques, parasites like Babesia spp., primarily recognized as animal pathogens, are becoming increasingly reported as human pathogens too, even in areas where the parasite has not been reported previously. Babesiosis should be included in the differential diagnoses for immunocompromised patients displaying fever worldwide.

\section{Acknowledgments}

We are thankful to Andrea Anda, Greta Strakl, Valerija Cvetko Weiss, Jozica Gruskovnjak, Martin Sagadin, Sabina Islamovic, and Nika Caf for their technical assistance.

This work was supported by the Slovenian Research Agency (grant no. P3-0083).

\section{About the Author}

Dr. Smrdel is a researcher at the Institute of Microbiology and Immunology, Faculty of Medicine, University of Slovenia, Ljubljana, Slovenia. Her research interests are babesiae and Anaplasma phagocytophilum.

\section{References}

1. Hildebrandt A, Gray JS, Hunfeld K-P. Human babesiosis in Europe: what clinicians need to know. Infection. 2013; 41:1057-72. http:/ / dx.doi.org/10.1007/s15010-013-0526-8

2. Krause PJ. Human babesiosis. Int J Parasitol. 2019;49:165-74. http:/ / dx.doi.org/10.1016/j.ijpara.2018.11.007

3. Olmeda AS, Armstrong PM, Rosenthal BM, Valladares B, del Castillo A, de Armas F, et al. A subtropical case of human babesiosis. Acta Trop. 1997;67:229-34. http:/ /dx.doi.org/10.1016/S0001-706X(97)00045-4

4. Schnittger L, Yin H, Gubbels MJ, Beyer D, Niemann S, Jongejan F, et al. Phylogeny of sheep and goat Theileria and Babesia parasites. Parasitol Res. 2003;91:398-406. http://dx.doi.org/10.1007/s00436-003-0979-2 
5. Ozubek S, Aktas M. Molecular evidence of a new Babesia sp. in goats. Vet Parasitol. 2017;233:1-8. http:/ /dx.doi.org/ 10.1016/j.vetpar.2016.11.016

6. Orkun Ö, Karaer Z. Molecular characterization of Babesia species in wild animals and their ticks in Turkey. Infect Genet Evol. 2017;55:8-13. http://dx.doi.org/10.1016/ j.meegid.2017.08.026

7. Hornok S, Takács N, Kontschán J, György Z, Micsutka A, Iceton S, et al. Diversity of Haemaphysalis-associated piroplasms of ruminants in Central-Eastern Europe, Hungary. Parasit Vectors. 2015;8:627. http:/ / dx.doi.org/ 10.1186/s13071-015-1236-0

8. Jia N, Zheng YC, Jiang JF, Jiang RR, Jiang BG, Wei R, et al. Human babesiosis caused by a Babesia crassa-like pathogen: a case series. Clin Infect Dis. 2018;67:1110-9. http://dx.doi.org/10.1093/cid/ciy212

Corresponding author: Tatjana Avsic-Zupanc, Institute of Microbiology and Immunology, Faculty of Medicine, Zaloska 4, SI-100 Ljubljana, Slovenia; email: tatjana.avsic@mf.uni-lj.si

\section{Hepatitis A Hospitalization Costs, United States, 2017}

\author{
Megan G. Hofmeister, Shaoman Yin, Maria V. Aslam, \\ Eyasu H. Teshale, Philip R. Spradling
}

Author affiliation: Centers for Disease Control and Prevention, Atlanta, Georgia, USA

DOI: https://doi.org/10.3201/eid2605.191224

The United States is in the midst of unprecedented person-to-person hepatitis A outbreaks. By using Healthcare Cost and Utilization Project data, we estimated the average costs per hepatitis A-related hospitalization in 2017. These estimates can guide investment in outbreak prevention efforts to stop the spread of this vaccine-preventable disease.

$\mathrm{T}$ The introduction of hepatitis A vaccine has dramatically changed the epidemiology of hepatitis A in the United States. After vaccine licensure in 1995, hepatitis A incidence declined substantially; 3,366 hepatitis A cases were reported nationally in 2017 (1).

During July 1, 2016-February 7, 2020, state health departments publicly reported $>31,000$ outbreak-associated cases, primarily affecting persons who use drugs and persons experiencing homelessness, in the largest person-to-person hepatitis A outbreaks in the postvaccine era (2). More than 18,900 (61\%) outbreakassociated patients have reportedly been hospitalized in these outbreaks (2). As these unprecedented outbreaks continue, we sought to estimate the average direct medical costs per hepatitis A-related hospitalization, which can be used to guide investment in outbreak prevention efforts.

We analyzed data from the 2017 Healthcare Cost and Utilization Project National Inpatient Sample (NIS). NIS, a $20 \%$ stratified sample of discharges from US community hospitals, is the largest publicly available all-payer inpatient database in the country (3). We considered a hospitalization to be hepatitis A-related if it included codes B15.0 or B15.9 from the International Classification of Diseases, 10th Revision, Clinical Modification, as any of the 30 listed diagnosis codes. We converted the total hospital charges into cost estimates (in 2017 US dollars) by multiplying total charges with 2017 hospital-specific cost-to-charge ratios (4), then estimated the average cost of hospitalization, SD, and 95\% CI on the basis of the NIS survey sampling design. We multiplied the average costs by the number of patients hospitalized for outbreak-associated hepatitis A to generate an estimate of the preventable economic burden of hospitalizations in the ongoing person-to-person outbreaks (2).

We examined hepatitis A-related hospitalizations in the 2017 NIS dataset for evidence of associated liver transplantation (procedure codes 0FY00Z0, 0FY00Z1, and 0FY00Z2 from the International Classification of Diseases, 10th Revision, Clinical Modification, listed as any of the 15 procedure codes). Because the unweighted number of hospitalizations associated with liver transplantation was $<10$, we included such hospitalizations in the analysis but did not report them as a separate category (5).

Overall, the average costs per hepatitis A-related hospitalization in the United States in 2017 were $\$ 16,232$ (SD $\$ 602 ; 95 \%$ CI $\$ 15,052-\$ 17,411$ ). The average costs ranged from $\$ 12,921$ (SD $\$ 1,443 ; 95 \%$ CI $\$ 10,091-\$ 15,750)$ in the West North Central Census Division to $\$ 19,680$ (SD $\$ 1,932 ; 95 \%$ CI $\$ 15,891-$ $\$ 23,467)$ in the Pacific Census Division.

During July 1, 2016-February 7, 2020, a total of 32 states reported $>18,900$ outbreak-associated hepatitis A hospitalizations resulting from the ongoing hepatitis A outbreaks (2). On the basis of results of our analysis as a multiplier, we estimate that hospitalization costs associated with these outbreaks have exceeded \$306.8 million (SD \$11.4 million) as of February $7,2020$. 